Conference Paper

\title{
A writing aid for dysgraphia affected people
}

Ahmed, W., Hasan, S., Shoaib, S., Houlden, N. and Nestiurkina, M.

This is a paper presented at 2020 IEEE NW Russia Young Researchers in Electrical and Electronic Engineering Conference, Moscow, Russia, 27-30 Jan. 2020

Copyright of the author(s). Reproduced here with their permission and the permission of the conference organisers.

\section{Recommended citation:}

Ahmed, W., Hasan, S., Shoaib, S., Houlden, N. and Nestiurkina, M. (2020) 'A writing aid for dysgraphia affected people.' In Proc. 2020 IEEE NW Russia Young Researchers in Electrical and Electronic Engineering Conference, Moscow, Russia, 27-30 Jan. 2020, pp. 2468-2471. doi 10.1109/EIConRus49466.2020.9039211 


\title{
A Writing Aid for Dysgraphia Affected People
}

\author{
Waqas Ahmed ${ }^{1}$, Shams-ul-Hasan ${ }^{1}$, Sultan Shoaib ${ }^{2}$, Nigel Houlden ${ }^{2}$, Mariia Nestiurkina ${ }^{3}$ \\ ${ }^{1}$ HITEC University, Taxila, Pakistan \\ ${ }^{2}$ Glyndwr University, Wrexham, UK \\ ${ }^{3}$ Moscow Institute of Electronic Technology, Moscow, Russia
}

\begin{abstract}
Dysgraphia is a writing disability due to ineffective working of motor neurons, causing the patient to face difficulty in writing. Many studies have been done to overcome Dysgraphia but unfortunately, only therapies are made instead of any aid. In this paper, a movement aid is proposed that removes the need of any therapy. The proposed methodology comprises of acquisition of hand movement signal from Motor Cortex and Sensory Brain area by using EEG scanning. These signals are then processed using Brain Computer Interface (BCI2000), which mainly includes Features selection, extraction and translational algorithms to convert signals into commands. These commands are then used to control Hand Movement device. The Hand Movement device uses the FES, applied at the forearm to perform different hand movements. This paper focuses on the flexion and extension of the fist.
\end{abstract}

Keywords-electroencephalogram; EEG; functional electrical stimulation; FES; brain computer interface; BCI; electrocorticogram, electromyogram; functional magnetic resonance imaging

\section{INTRODUCTION}

Dysgraphia is a learning disability where the standard of handwriting is lower than required for the classified age, calibre and academic standard. This is a hereditary disease which is mainly categorized into two classes: perceptual and motor. The first one is associated with the failed linkage of symbols and signs with the spelling of the word depicting the sound. While in the latter one, the patient can read and speak the words properly but lacks motor skills causing hindrance in writing. Hence evaluating that motor dysgraphia occurs due to improper functioning of motor neurons, causing the patient to face difficulty in writing. Considering the problem, the later one is the most prevalent one and has the percentage of dysgraphia-effected people all across the World has raised from $21 \%$ to $33 \%$ in 2011 . This research work aims to improve the motor dysgraphia by fabricating a device that assists in the natural movement of hand through brain signals. Many approaches have been proposed in the literature of which there are two most prevalent techniques. The first technique consists of special-purpose applications and exercises like joining the dots, to make patients write a word. The second consists of different therapies which mainly include Occupational Therapy and Educational Therapy. The results obtained from these therapies are not up to the mark and involve muscle fatigue and costly hectic routines ranging from $\$ 300-\$ 400$ per hour and costs tend to be on the higher end depending upon the condition of the patient and hospital type.

To overcome all these issues, a movement aid is proposed which does not require any therapy at all and assists the patients to write properly and perform all the movements. The device can be used as a regular wearable device for 20 consecutive hours without any muscle fatigue. The proposed methodology comprises of acquisition of hand movement signal from the Motor Cortex and Sensory Brain area using electroencephalogram (EEG) scanning [1]. These signals will be processed using different engineering techniques to carry out required FES voltage patterns. These patterns will then be fed to a hand movement instrument which will stimulate the forearm muscles to carry out all types of finger movements.

As a large proportion of the world's population is suffering from this disability, this product will highly serve the dysgraphia patients not only in Pakistan but across the world. This product will also reduce/eliminate the dependability of the patients on others. Also, the study will open new doors towards the Brain Computer Interface (BCI) based devices for impaired people not only with dysgraphia but many others which are present in this world waiting to be addressed.

\section{LITERATURE REVIEW}

Xiao et al. [2] used EEG to distinguish between four motor imagery (MI) types. Principal Component Analysis and squared cross-correlations were administered for deriving the spatial and spectral characteristics: analyzed by linear discriminant analysis (LDA) classifier. Lu et al. [3] studied BCI depending on EEG constitutes of Movement Related Potentials (MRPs) feature; which is challenging to derive as it is noisy and alters person to person. Adaptive Spatio-Temporal (AST) filtering technique was proposed to construct MRPs precisely in lesser space capacity where Gaussian kernel is administered, enhancing all parameters. Liu et al. [4] investigated ipsilateral cortical signals using EcoG, translating finger articulations administering machine learning techniques. The outcomes were successful depicting great accuracies in every case by only taking a fraction of features captured. Xiao et al. [5] proposed distinguishing the finger movements using non-invasive EEG instead of ECoG through spectral principal component analysis (PCA). Hence, the enhancing number of features for control, assisting in the advancement of noninvasive BCI. Shakeel et al. [6] paraphrased certain characteristics such as attaining signal, sorting, improving and different electrode assortments administered through EEG redesigning multifarious investigations using MRCPs to anticipate the imagined movement. Patel [7] established different algorithms to monitor hand articulation through EEG by BCI applications. This technique was applied to two electrodes minimizing the workload and attaining better accuracy. The EEG signals were passed through bandpass filter; minimizing noise and spatial filter, minimizing noise in 
transmission. Liang and Bougrain [8] proposed that signal processing is vital to convert brain signals into commands through BCI. So, a technique was formulated modulating the amplitude of band-specific ECoG accompanied by a limited memory for anticipating finger movement. The analogy between the predicted and recorded movements proved a high success rate. Xiao and Ding [9] applied spectral principal component analysis (PCA) on EEG signals acquired by moving a finger to verify cross-frequency spectral structures. These were analysed in spatial patterns, cross condition pattern changes, identifying the potential of finger articulation from rest and translation of each finger movement in contrast to classic mu/beta rhythms. Oliveria et al. [10] proposed a methodology to detect and omit bad channels and individual components of EEG data by template correlation rejection (TCR) having noise in motion. An analysis is carried out by comparing it with the template designed. Jochumsen et al. [11] compared the techniques of identifying and classifying of MRCPs through EEG of healthy and stroke patients. Templates comparing approach accompanied by a set of spatial filters were administered from MRCPs of four hinted tasks and employing the combination of temporal, spectral, timescale, or entropy-based features. Wilson et al. [12] proposed that BCI was used to detect and translate EEG signals by wearing EEG electrode pads on the scalp. A test subject was analysed by performing different articulations of hand and feet, and best channels were marked for determining a significant change in mu and beta rhythms of the brain. Morash et al. [13] investigated neural signals prior to articulations and motor imagery for anticipating, which motor imageries is about to happen, accessing this through BCI applications. Pfurtscheller et al. [14] studied BCI to monitor Functional Electrical Stimulation (FES). These stimulations were received in one of the EEG channels, which showed that pulse was generated when stimulus increased the given limit resulting in hand movement. Muralidharan et al. [15] used EEG to record stimulus of a paralyzed hand. He gave electrical impulses to the subject through a device to monitor the opening and closing of a paralyzed hand and altered the threshold of the device for smooth movement. Fifer et al. [16] used intracranial electroencephalographic (iEEG) signals to detect the reaching and grasping movement made by the subject in order to recognize the specific electrodes attached to the subject's hand. Tamaki et al. [17] made a device that controls a human hand using electrical pulses. The device basically stimulates the actuating muscles of the human forehand. All the stimulation controlling is done by a device worn on the forearm. Muller et al. [18] illustrated wrist movements through EEG by FES. He suggested that these induced movements were somehow similar to natural hand movements. Giordano and Maiorana [19] developed software in order to help dysgraphia affected patients. The software basically uses JavaScript libraries and gesture recognition algorithms. It assists the subjects to carry out various exercises of handwriting like joining the dots to complete an alphabet. Chestek et al. [20] investigated if the impulses by FES provided ample information to ECoG of varying hand postures. Mangold et al. [21] studied different cases by providing them with the treatment of FES for hand movement and grasping. He carried out various tests on different subjects to attain results regarding the positive and negative outcomes of this technology. The results proved that further enhancement was required to fulfil the limitations but this proved an efficient tool for natural hand movement.

\section{IMPLEMENTATION PROCEDURE}

In order to make our system familiar with EEG signals, we first have to process the EEG signals to obtain a full data set. As discussed earlier, the human brain emits EEG signals at continually. Those signals vary based on frequency and origin of emission. Before using those signals, one has to decide which frequency (rhythm) is required to have the particular information, in our case we are looking for $\mathrm{Mu} / \mathrm{beta}$ rhythm (as all motor-related frequencies are present in this range). However, the problem still remains of which electrode should be considered and which should be ignored for EEG signal acquisition. This problem is countered by Feature selection. Features are valuable information that is extracted from a signal. In case of EEG signal, we had to locate the position and frequency for an appropriate signal which is known as feature selection.

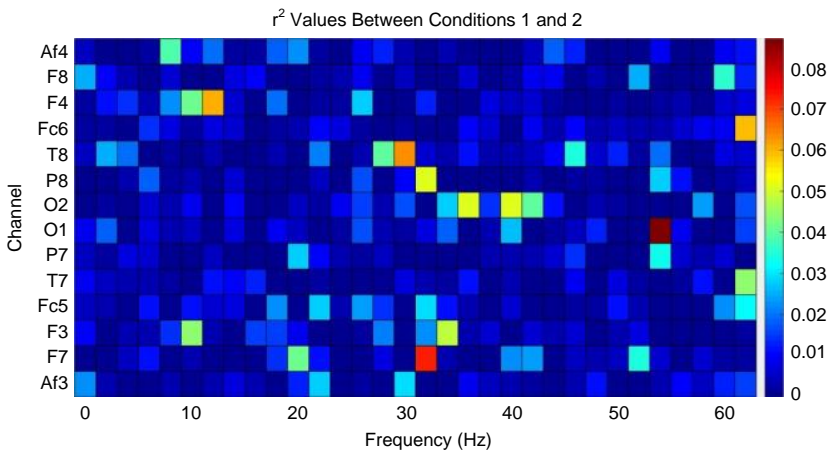

Fig. 1. Feature map for both hands.

To perform feature selection, we first need to have a feature map which was generated using BCI2000 by using its built-in stimulus functionality. The stimulus functionality gave us detailed information regarding frequency and power of the evoked signal with respect to movement performed. To carry out stimulus presentation, it is necessary to have an isolated environment to have as minimum artefacts as possible. Separate view (screens) for both subject and examiner was used in order to provide both of them space to be comfortable.
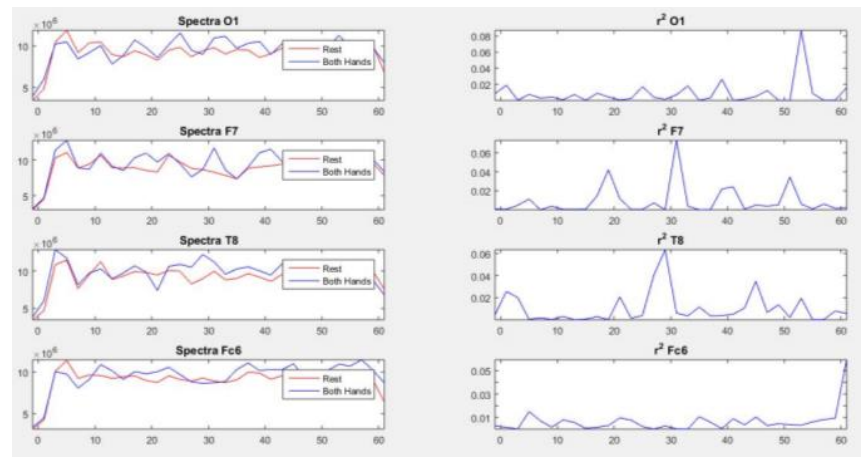

Fig. 2. Both hands normalized r-squared

BCI2000 does not support Emotiv Epoc out of the box. We had to use its drivers contributed by Dr. Griffin Milsap. Once the driver was build any ready we launched the BCI2000 and 
set all the required parameters with zero filtering (as we need to have all the signals at all frequencies). After Parameter setting the stimulus session was started, and the acquired signal was recorded. During the session, different instructions were shown to the subject followed by rest intervals. The subject was asked to perform or imagine movement related to those instructions. After recording a sufficient amount of sessions, the recorded data were analysed using Offline Analysis Tool. The tool gave us the Feature maps for performed actions (Fig. 1).

The above figure illustrates the engagement of both hands in the recorded sessions. On the $x$-axis we have Frequency and on the $y$-axis we have a respective channel. By this map, we can easily find the required signal at the required electrode. The Offline analysis tool also generates graphs showing normalized $\mathrm{r}^{2}$ values and scalp graphs for better understanding. Both types of graphs for the above mention feature maps are given in Fig. 2.
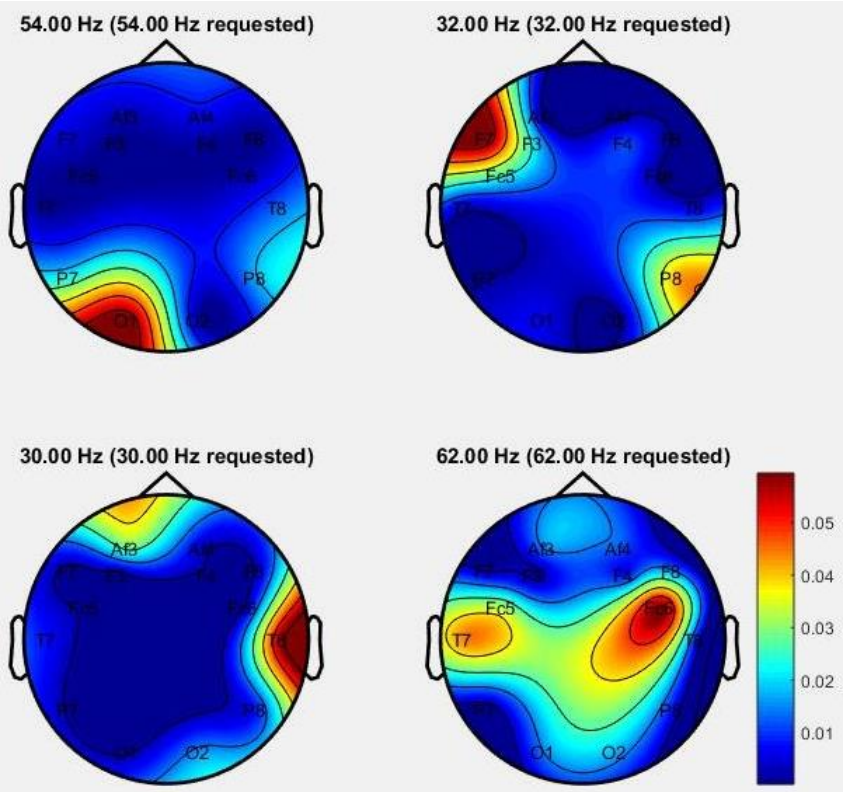

Fig. 3. Scalp presentation for both hands.

After gathering this information, we used it to perform cursor task, a built-in application to check that the steps described above are done and working properly or not. We launched the BCI2000 with appropriate parameters and filters (CAR) to remove artefacts and to suppress all other incoming signals except the required. The Cursor moves vertically up and down with the movement of the subject's hands and moves downwards at rest. The subject is asked to make the ball hit the target.

The main concept is calculating $\mathrm{r}^{2}$ values at each interval. If the value of the selected electrode at the selected frequency is greater than the threshold, represents movement and below threshold represents rest.

\section{SYSTEM ARCHITECTURE}

The device circuit constitutes of a variable, power supply, pulse generator, switching series (AQV 221 PhotoMOS Relays) and microcontroller Arduino mega 2560, 28 electrode pads on two belts. The electrode pads used are self-stick Tyco gel Carbon electrode pads of size $10 \times 30 \mathrm{~mm}$ as it causes no pain even at stimulation level of $33 \mathrm{~V}$. The $\mathrm{J} 114$ pin connector connects to the micro-controller that controls the switching of the AQV221 PhotoMOS-relays. The pulse generator section consists of a 555 timer set at astable-mode to produce a square pulse of $40 \mathrm{~Hz}$ and of for $0.2 \mathrm{~ms}$. The pulse is then inverted using the 74LS14 to produce the required pulse, which is on for $0.2 \mathrm{~ms}$. This pulse is then amplified to the required level using the LM358 single supply op-amp. The output is then fed to the switching section made of AQV221 PhotoMOS-relays. The positive electrode pads are connected to the output of each relay independently using the 14 pin connector $\mathrm{J} 2$. The ground electrode pads are connected to another 14 pin connector which is connected to the ground of the whole circuit. The solid-state relays or semiconductor relays offer better solutions for high precision applications than the electromechanical relays.

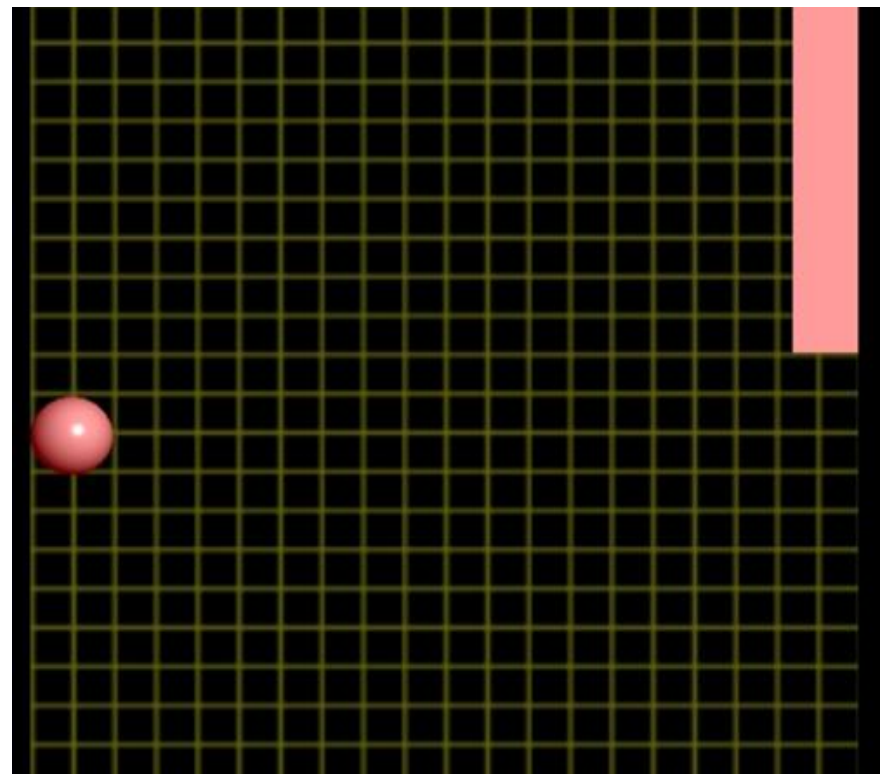

Fig. 4. Cursor movement.

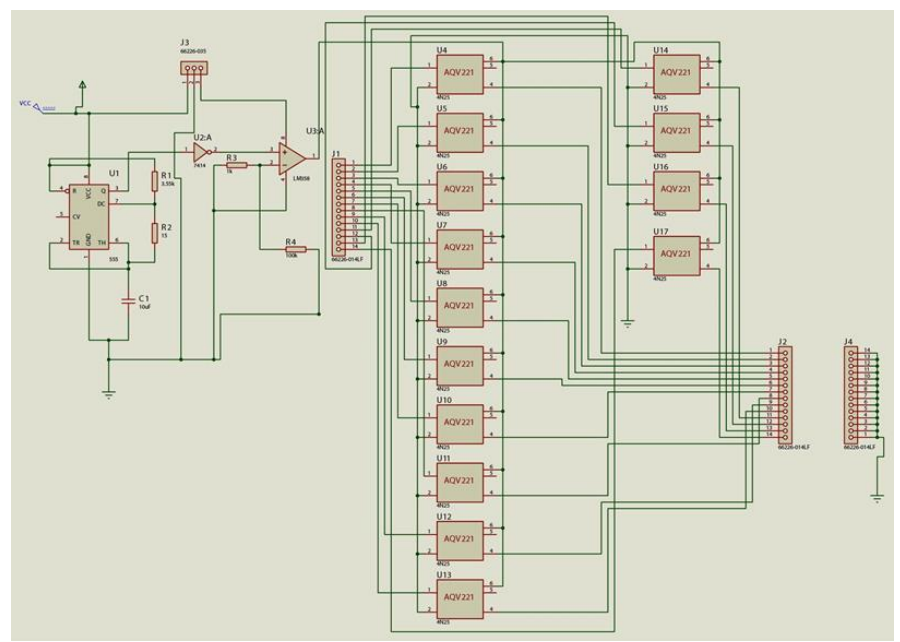

Fig. 5. Circuit diagram of the complete system. 


\section{CONCLUSION}

This paper presented a writing aid for dysgraphia affected people using brain computer interface. All the subjects were non-familiar to BCI and never used it before undergoing this study. The BCI was easily adapted by all the subjects in the limited number of trails and sessions. The most promising thing in BCI is that subject does not have to actually perform the movement instead he has to imagine it and the same behaviour is observed in EEG which means the device can not only be used to help dysgraphia affected people but people having hand movement impurities can also be benefitted. The subject acclimatised to use very rapidly and after some time they reported that they are not concentrating on moving hand but moving the cursor. The overall efficiency of the system is calculated to be approx. $75 \%$. The efficiency can be increased by increasing number of electrodes over the motor cortex and sensory brain. The overall results are very much satisfactory and can pave a way to further research in this domain. This research paper is limited to only fist extension and flexion due to limited amount of time and resources. But in the future, it can be extended to complete fingers movement and control.

\section{ACKNOWLEDGMENT}

We would like to thank Engr. Shahid Latif for his help and guidance in electronics parts. We would also like to thank Dr. Griffin Milsap for his precious time in helping us in the need of the hour. Lastly, we would like to thank Engr. Iftikhar Ahmed and Engr. Riaz for their guidance and support.

\section{REFERENCES}

[1] G. Acar, O. Ozturk, A.J. Golparvar, T.A. Elboshra, K. Bohringer, and M.K. Yapici, "Wearable and flexible textile electrodes for biopotential signal monitoring: A review," Electronics, vol. 8, no. 5, article 479, May 2019.

[2] R. Xiao, K. Liao, and L. Ding, "Discriminating multiple motor imageries of human hands using EEG," in Proc. IEEE Ann. Int. Conf. on Engineering in Medicine and Biology Society (EMBC), 28 Aug. - 1 Sept. 2012, San Diego, USA, pp. 1773-1776.

[3] J. Lu, K. Xie, and D.J. McFarland, "Adaptive spatio-temporal filtering for movement related potentials in EEG-based brain-computer interfaces." IEEE Transactions on Neural Systems and Rehabilitation Engineering, vol. 22, no. 4, pp. 847-857, July 2014.

[4] R. Xiao, and L. Ding, "Classification of finger pairs from one hand based on spectral features in human EEG," in Proc. IEEE Ann. Int. Conf. on Engineering in Medicine and Biology Society (EMBC), 26-30 Aug. 2014, Chicago, USA, pp. 1263-1266.

[5] Y. Liu, M. Sharma, C.M. Gaona, et al., "Decoding ipsilateral finger movements from ECoG signals in humans," in Proc. 23rd Int. Conf. on Neural Information Processing Systems, 6-9 Dec. 2010, Vancouver, Canada, pp. 1468-1476.

[6] A. Shakeel, M.S. Navid, M.N. Anwar, S. Mazhar, M. Jochumsen, and I.K. Niazi, "A review of techniques for detection of movement intention using movement-related cortical potentials," Computational and Mathematical Methods in Medicine, vol. 2015, article 346217, 2015.
[7] N.D. Patel, "An EEG-based dual-channel Imaginary motion classification for brain computer interface," Dissertation, Lamar University, Lamar, USA, 2011.

[8] N. Liang, and L. Bougrain, "Decoding finger flexion from band-specific ECoG signals in humans," Frontiers in Neuroscience, vol. 6, article 91, June 2012.

[9] R. Xiao, and L. Ding, "EEG resolutions in detecting and decoding finger movements from spectral analysis," Frontiers in Neuroscience, vol. 9, article 308, Sept. 2015.

[10] A.S. Oliveira, B.R. Schlink, W.D. Hairston, P. Konig, and D.P. Ferris, "A channel rejection method for attenuating motion-related artefacts in EEG recordings during walking," Frontiers in Neuroscience, vol. 11, article 225, April 2017.

[11] M. Jochumsen, I.K. Niazi, N. Mrachacz-Kersting, N. Jiang, D. Farina, and K. Dremstrup, "Comparison of spatial filters and features for the detection and classification of movement-related cortical potentials in healthy individuals and stroke patients," Journal of Neural Engineering, vol. 12, no. 5, article 056003, Oct. 2015.

[12] J.A. Wilson, G. Schalk, L.M.Walton, and J.C. Williams, "Using an EEG-based brain-computer interface for virtual cursor movement with BCI2000," Journal of Visualized Experiments: JoVE, vol. 29, article 1319, 2009.

[13] V. Morash, O. Bai, S. Furlani, P. Lin, and M. Hallett, "Classifying EEG signals preceding right hand, left hand, tongue, and right foot movements and motor imageries," Clinical Neurophysiology, vol. 119, no.11, pp. 2570-2578, Nov. 2008.

[14] G. Pfurtscheller, C. Brunner, A. Schlogl, and F.H.L. da Silva, "Mu rhythm (de) synchronization and EEG single-trial classification of different motor imagery tasks," NeuroImage, vol. 31, no.1, pp. 153-159, May 2006.

[15] A. Muralidharan, J. Chae, and D.M. Taylor, "Extracting attempted hand movements from EEGs in people with complete hand paralysis following stroke," Frontiers in Neuroscience, vol. 5, article 39, 2011.

[16] M.S. Fifer, G. Hotson, B.A. Wester, et al., "Simultaneous neural control of simple reaching and grasping with the modular prosthetic limb using intracranial EEG," IEEE Transactions on Neural Systems and Rehabilitation Engineering, vol. 22, no. 3, pp. 695-705, Oct. 2013.

[17] E. Tamaki, T. Miyaki, and J. Rekimoto, "PossessedHand: A hand gesture manipulation system using electrical stimuli," in Proc. 1st Augmented Human International Conf., 2-3 April 2010, Megeve, France, article. 2.

[18] G.R. Muller, C. Neuper, R. Rupp, C. Keinrath, H.J. Gerner, and G. Pfurtscheller, "Event-related beta EEG changes during wrist movements induced by functional electrical stimulation of forearm muscles in man," Neuroscience Letters, vol. 340, no. 2, pp. 143-147, April 2003.

[19] D. Giordano, and F. Maiorana, "Addressing dysgraphia with a mobile, web-based software with interactive feedback," in Proc. IEEE-EMBS Int. Conf. on Biomedical and Health Informatics (BHI), 1-4 June 2014, Valencia, Spain, pp. 264-268.

[20] C.A. Chestek, V. Gilja, C.H. Blabe, B.L. Foster, K.V. Shenoy, J. Parvizi, and J.M. Henderson, "Hand posture classification using electrocorticography signals in the gamma band over human sensorimotor brain areas," Journal of Neural Engineering, vol. 10, no. 2, article 026002, April 2013.

[21] S. Mangold, T. Keller, A. Curt, and V. Dietz, "Transcutaneous functional electrical stimulation for grasping in subjects with cervical spinal cord injury," Spinal Cord, vol. 43, no. 1, pp. 1-13, Jan 2005. 